\title{
TRANSFER OF LOW-POTENTIAL HEAT FROM A DEEP BOREHOLE BY MEANS OF A HEAT PIPE AND HEAT PUMP
}

The paper presents a design of equipment for the usage of low-potential heat collected from rock in a deep borehole by means of heat pipes (HP) and a description of its realization. The borehole model together with a CFD simulation of heat transfer from rock to a soil probe enabled dimensioning of a heat exchanger on the condenser part of the heat pipe and choice of a suitable heat pipe charge. Part of the paper deals with the processing of measurement results gathered from the comparative experimental equipment requiring forced and unforced circulation(heat pipe) of the working substance on the primary side of the heat pump.

Keywords: Heat pipe, heat pump, low-potential heatofthe rock,output, deep borehole, soil probe.

\section{Introduction}

Low-potential heat of the rock is a heat flow in the surface area of the Earth crust with a relatively low thermal potential corresponding to the standard geothermal gradient. This heat flow can either originate from solar radiation falling on the Earth surface or from radiogenic processes inside the Earth.

The heat falling on the Earth surface due to solar radiation is timely highly unstable and influences the heat flow in the Earth rocks up to the depth ranging from 10 to 20 meters. Yearly and daily changes in solar radiation intensity and outer air temperature cause temperature variations in the thin layer of the Earth's surface. Heat regime in the depth greater than the depth of the neutral zone (in Slovakia approx. $14 \mathrm{~m}$ ), is formed due to the effects of heat characteristics of the Earth's core. The flow of radiogenic heat produced inside the Earth is practically stable and is not influenced by any seasonal or other changes of weather and temperature. The temperature of the Earth increases with the depth from the surface in compliance with the geothermic gradient of a particular geological structure (approximately $3{ }^{\circ} \mathrm{C}$ for every $100 \mathrm{~m}$ ). The amount of radiogenic heat from inside the Earth differs for different areas of the Earth crust. The heat flow for Central Europe ranges from 0.05 to $0.12 \mathrm{~W} \cdot \mathrm{m}^{-2}$.

A great advantage of low-potential geothermal heat is that it is not limited by a geothermal region or any region with a high potential of hot geothermal waters. It can theoretically be recovered anywhere on Earth's surface. It is the heat accumulated in the soil and ground waters. Rock temperatures range from $5{ }^{\circ} \mathrm{C}$ to $60{ }^{\circ} \mathrm{C}$. Heat can be collected directly from the soil by means soil collec- tors, from boreholes up to the depth of $400 \mathrm{~m}$ or from ground waters. Low-potential heat (up to $60^{\circ} \mathrm{C}$ ) can be used to heat buildings, water, playgrounds, etc.Compared with high-potential heat the costs for low-potential heat collection are considerably lower.

When using low-potential geothermal energy there are two basic systems - the system with a forced circulation of a heat carrying medium or the system with thermosiphoneffect without a forced convection in a ground heat exchanger. In both systems the heat pump compressor circulation is predominantly used for the heat potential transformation [1], [2].

Heat carrying substances used for forced circulations in the heat pump primary circuit are usually mixtures of water and antifreeze, such as, e.g. ethylene glycol, tyfocor, salt brine, etc. Owing to the fact that relatively efficient circulation pumps need electricity for their operation, new ways of heat transfer without a forced circulation of heat carrying medium in the ground heat exchanger are looked for. Heat pipes with suitable charge seem to be a perspective way of collecting heat from rocks without a forced circulation of a working substance. In such a system the heat carrier flows in the pipe without additional energy supply with small temperature difference making use of the properties of heat transfer at phase changes. The device working on the mentioned principle of heat transfer is called a heat pipe. Heat pipes have been recently commonly used for the cooling ofelectronic components and electronic devices (processors, transformers, etc.) and are examples of real usage of heat transport without a forced circulation of a heat carrier. In heat technology heat pipes are used also for solar energy transfer in high-efficiency solar collectors and in systems for heat recovery.

\footnotetext{
* Milan Malcho, Jozef Jandacka, Martin Vantuch, Andrej Kapjor, Stanislav Gavlas

Department of Energy Technology,Faculty of Mechanical Engineering, University of Zilina, Slovakia, E-mail: milan.malcho@fstroj.uniza.sk
} 


\section{A design of device for low-potential heat collection}

The device for low-potential heat collection from the rock in a deep borehole was realized by means of a conventional and unconventional way using heat pumps [3]. Due to the fact that there are two independent soil probes making use of two different ways of heat collection, we used two heat pumps to extract the heat (abbr. HP) of the type Ground/Water working independently from each other Fig. 1. The whole device together with all the measuring systems (with the exception of deep boreholes) was placed in laboratory premises. The pipes of primary circuit were led to the boreholes located in front of the laboratory building.

The whole device thus consists of two heat pumps connected through the primary circuit to two boreholes 150 meters deep drilled in the vicinity of Zilina University laboratories. The boreholes were performed by means of a drilling set equipped with a collapsible drilling tower with a grief stem, rotary table, and its own power unit (diesel engine). This drilling set is designed for engineering and geological boreholes and boreholes to extract heat from soil.

To avoid mutual thermal influencing of the boreholes, there is a distance of 14 meters between them. In the boreholes there are groundU-heat exchangers using two different ways of heat collection from soil:

- with a forced circulation of the working medium in the primary circuit (soil probe),

- with a natural thermosiphon circulation of the working medium in the primary circuit (heat pipe).

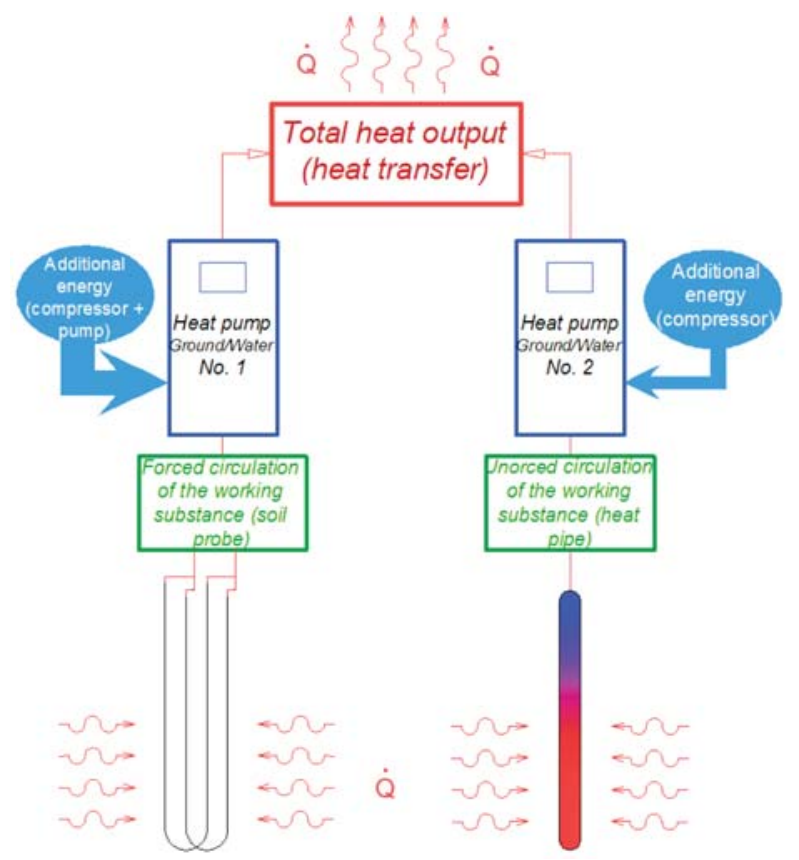

Fig.1 Scheme of experimental device for low-potential heat collection from deep borehole

\section{A design of the heat pipe}

\subsection{Numerical model of heat transfer from the rock into the heat pipe}

As the structure of the rock changes with the depths, its thermokineticcharacteristics also change. The process of the rock cooling will, therefore, be modeled according to various layers with different thermokinetic characteristics of the given layer of a 150 meter borehole.

Heat transfer from the rock into the heat pipe is described by Fourier's equation for heat conduction in cylindrical coordinates

$$
\rho c_{p} \frac{\partial t}{\partial \tau}=\frac{\partial}{\partial x}\left(\lambda \frac{\partial t}{\partial x}\right)+\frac{\partial}{\partial y}\left(\lambda \frac{\partial t}{\partial y}\right)+\frac{\partial}{\partial z}\left(\lambda \frac{\partial t}{\partial z}\right)+q_{v}
$$

Supposing that physical characteristics of a body are constant, we can write relation (1) in the form

$$
\frac{\partial t}{\partial \tau}=\frac{\lambda}{c_{p} \rho}\left(\frac{\partial^{2} t}{\partial r^{2}}+\frac{1}{r} \frac{\partial t}{\partial r}+\frac{1}{r^{2}} \frac{\partial^{2} t}{\partial \varphi^{2}}+\frac{\partial^{2} t}{\partial z^{2}}\right)+\frac{q_{V}}{c_{p} \rho} .
$$

Temperature in the cylinder axis can be written as

$$
t(r, \tau)=t_{0}+\frac{q_{t}}{4 \pi \lambda} \int_{0}^{\tau} \frac{e \frac{-r^{2}}{4 a \tau}}{t} d t=t_{0}+\frac{q_{t}}{4 \pi \lambda} W(u),
$$

where the indefinite integral can be expressed in the form of infinite series by means of a substitution

$$
u=\frac{r^{2}}{4 a \tau}
$$

as

$$
\begin{aligned}
& W(u)=-\gamma-\ln u+u- \\
& -\frac{u^{2}}{2.2 !}+\frac{u^{3}}{3.3 !}+\frac{u^{4}}{4.4 !}+\ldots
\end{aligned}
$$

where

$\gamma \quad$ is Euler-Mascheroni constant (0.5772).

The numerical model was set up on the basis of experimentally determined input thermokinetic parameters of the rock and boundary conditions with an objective to create the situation similar to real conditions. The objective of heat transfer simulation in the observed borehole was to determine the temperature field and heat flows in the rock in dependence on the borehole depth. The numerical model was set up in the program ANSYS Workbench - Fluent [4]. This CFD software serves the simulation of thermophysical and physical phenomena in a wide range of software simulations. Mathematical modeling of concrete physical phenomena is enabled through fundamental physical models and equations. 
The course of temperature in the calculated temperature field near the borehole surface, mainly in the area of the probe output shows the drop of the temperature of medium at the output from the initial $4.8^{\circ} \mathrm{C}$ to $1.8^{\circ} \mathrm{C}$ after 22 days of heat collection simulation. The obtained heat output decreased with the increasing number of days Fig. 2. Its decrease depends on more parameters, but mostly on thermal conductivity and specific thermal capacity of the rock that are given as initial conditions. Numerical simulations showed that relevant data about thermokinetic parameters of the rock are needed for the exact simulation of heat collection from the rock in the vicinity of the deep borehole Fig. 3 .

Numerical simulation illustrates very well changes of thermal fields in the rock massif, mainly temperature changes in dependence on the borehole depth and time at heat collection. Temperature changes in the borehole were decisive for an analysis of a possible change in the behavior of heat pipe working substance at heat collection and subsequent decrease of heat potential of the lower part of the borehole.

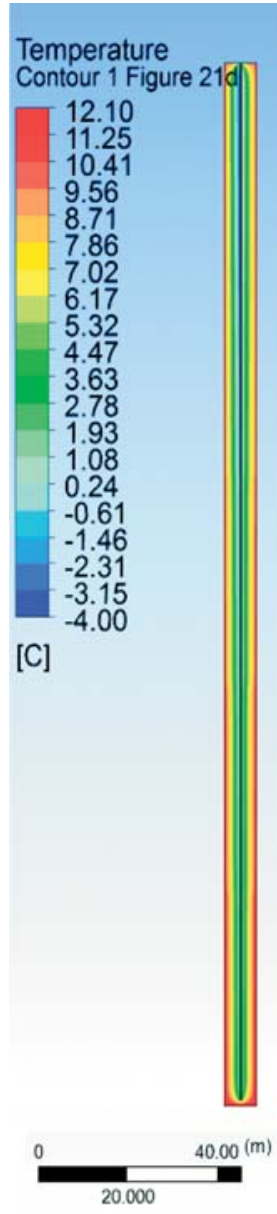

Fig. 2 Temperature field in the vicinity of the borehole after 21st day of heat collection

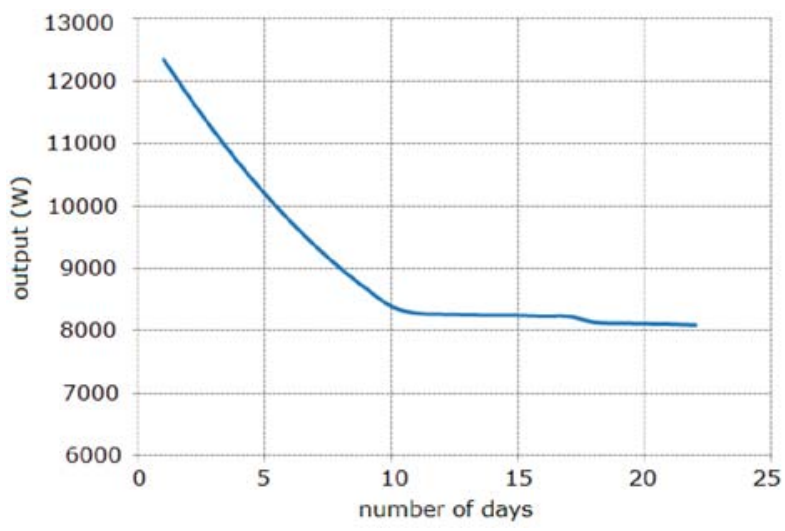

Fig. 3 Course of output obtained from borehole simulation

\section{Measurement of heat pipes output parameters}

The measurement was made after seven days when the temperatures in the borehole had been leveled off. The reason was to eliminate the influence of supplied ammonia on temperature changes in the deep borehole when the heat pipe was applied. During these seven days the heat pump was off and, consequently, the heat transfer from the borehole was stopped.

Due to the fact that the measurement was made in spring months which were extraordinarily warm, the secondary circuit of the heat pump had to be connected to the cooling tower to provide efficient heat removal on the secondary side of the heat pump. The continuous operation of the heat pump started when a stable appliance was connected to the heating (secondary) circuit.

Before the measurement $700 \mathrm{~g}$ of gaseous ammonia was removed from the heat pipe to avoid residual ballast gas and impurities in the upper condensation part of the heat pipe. During ammonia inlet, the heat pump operated at full output. When the heat pump was launched and ammonia removed, the process of phase change in the heat pipes immediately began. Immediate drop of temperatures in the heat pipe along the all depth of the borehole was recorded. The temperature decrease was recorded also in the next hours and also in the following day. During the heat pump operation the temperatures were decreasing nonlinearly mainly in the upper part of the heat pipe in the vicinity of the condensation heat exchanger. In these parts the temperature drop was the most intensive. The temperature in the depth of 5 meter dropped to the lowest minimum during the measurements, namely to $3{ }^{\circ} \mathrm{C}$. Considerable temperature drop was recorded up to the depth of $100 \mathrm{~m}$. The lowest temperature drop was recorded at the depth of 150 meters Fig. 4.

The efficiency of heat pipe was manifested on the temperature gradient of the heat pump primary circuit. At the start of measurement its difference was as many as $3.5^{\circ} \mathrm{C}$. Gradually, the temperature difference was mildly decreasing and at the end of measurement achieved $2.3{ }^{\circ} \mathrm{C}$, which was seen also in gradual decrease of heat output obtained from the borehole by means of the heat pipe Fig. 5 . 


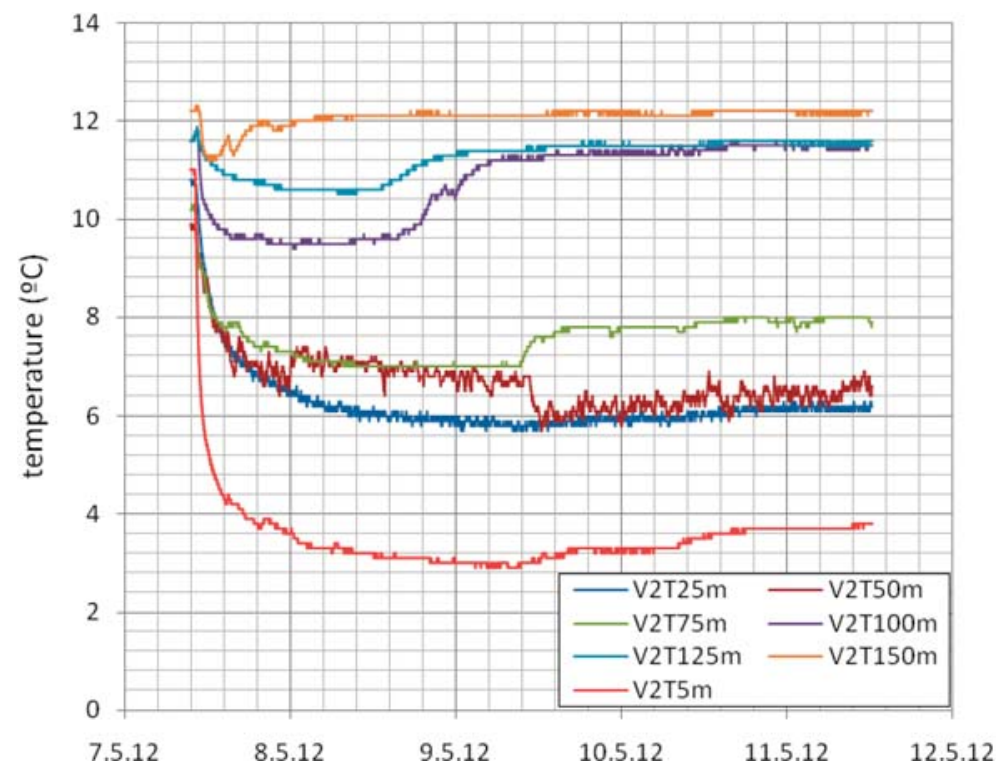

Fig. 4 Course of temperature changes in the borehole depths during heat pipe measurement.

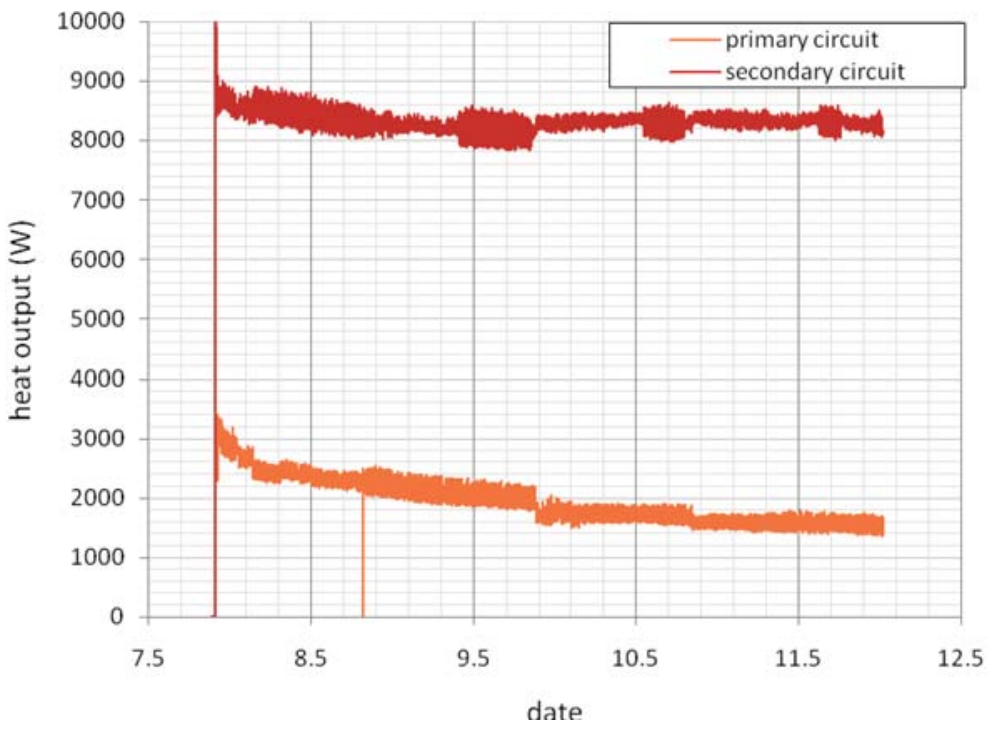

Fig. 5 Course of output change on the heat pump primary circuit

Resultant heat output measured on the heat pump primary circuit

\begin{tabular}{|l|l|}
\hline Max. output* & $3200 \mathrm{~W}$ \\
\hline Min. output* & $1550 \mathrm{~W}$ \\
\hline Average output* & $1900 \mathrm{~W}$ \\
\hline Average overpressure in HP & $3.42 \mathrm{Bar}$ \\
\hline
\end{tabular}

* - heat output obtained from the soil during measurement take on the primary side of heat pump

Table1
The difference between the output obtained by a classical method of a forced circulation of the working substance and the one obtained by the heat pipe was considerableTable1. It is obvious that the heat pipe did not continuously work with the designed heat transfer [5]. This fact can be attributed to more factors having influence on the correct operation of the heat pipe. One problem was removed at the introductory measurement of the heat pipe and the output considerably increased and the heat pipe began working. 


\section{Conclusion}

During the measurements taken one the heat pipe, more changes occurred and were manifested in the records of temperatures and output. One of them was a mild decrease of obtained output during all the measurements on the heat pipe. After a few days of measurements gradual leveling off the temperatures to the original value in greater depths was also recorded. There can be several causes of these facts:

- the heat pump was connected to the hot-air unit in the corridors of the building where was a surplus heat rejection. As the outer daily temperatures approached $28{ }^{\circ} \mathrm{C}$, the heat pump control more often turned on and off in short time intervals. This could result in continuous interruption of the process of phase change in the heat pipe which led to heat output drop. This problem was solved through stationary cooling but the output increase was not significant.

- Another possibility which might occur in our case may be unequal dosing of the working substance for individual $U$ tubes of the heat pipe. As the heat pipe consists of two independent U-tubes, the condensate dosing in the exchanger part can notbe influenced and it is impossible to provide and equal supply of the working substance into each of the U-tubes. This fact can cause that the condensate from the exchanger part leading to the lower part of the heat pipes flows to one U-tube only. It can result into the heat pipe output drop due to:

- loss of efficiency of one of the U-tubes (as it is empty there is no phase change in it),

- increases of pressure caused by the height of ammonia column in the full U-tube (it could, in the case of complete accumulation in one of the U-tubes, achieve the height of $60 \mathrm{~m}$ ). Hydrostatic pressure will increase and the total pressure in theheatpipewillincreaseaswell. Ifthiscaseoccurred, the liquid ammonia boiling would be limited by the depth in which the pressure would not exceed the boiling point at the given temperature.

\section{References}

[1] KAPJOR, A., HUZVAR, J., PILAT, P.: Effect of Operating Conditionsto a Temperaturein the Heat Pump Compressor (in Slovak), Erin 2011, 5th annual intern. travelling conference for young researchers and PhD students, Vysoké Tatry, 2011.

[2] LENHARD, R., JANDACKA, J., JAKUBSKY, M.: Devices for Simulating Low-potential Transformation of Geothermal Heat to Hheat Suitable for Heating (in Slovak), Zilina 2010.

[3] PAPUCIK, S., VANTUCH, M., JANDACKA, J.: Operating Measurement of Heat Pumps Air/Water (in Slovak), Vetranie a klimatizacia 2011: Systemy vetrania a klimatizacie v budovach s nizkou spotrebou energie, Tatranska Lomnica, 2011.

[4] LENHARD, R.: Numerical Simulation Device for the Transport of Geothermal Heat with Forced Circulation of Media, Fourth Global Conference on PCO 2010, Kuching - Sarawak - Malaysia 2010.

[5] CAJA, A., NEMEC, P., MALCHO, M.: The Dependence of Quantum and Filling Type to Heat Transport of Gravity Heat Pipe, Experimental fluid mechanics 2010, Liberec 2010. 